\title{
Pacientes con malestar en atención primaria: un instrumento de rastreo
}

Measuring patient distress in primary care.

Seelert K R, Hill R, Rogdon M, Schwenzfeier E. Fam Med 1999; 31 (7): 483-7

\section{Objetivo}

Evaluar la capacidad de un nuevo cuestionario (0utcome Questionnaire Short Form [0Q-10]), para detectar malestar psicológico (principalmente relacionado a ansiedad o depresión) en atención primaria. Evaluar cual es el puntaje útil en el que el 00-10 identifica los pacientes que requieren atención adicional por un especialista.

\section{Diseño}

Estudio de corte tranversal.*

\section{Lugar}

Consultorios de atención ambulatoria, Universidad de Utah, EE.UU.

\section{Pacientes}

Se incluyeron 292 adultos > 18 años en seguimiento ambulatorio que consultaron durante un período de 2 meses, en forma programada, a los médicos de planta y residentes de medicina familiar. Se excluyeron aquellos que estaban citados para consulta psicológica o test de laboratorio.

\section{Descripción del test y del Test de referencia}

El cuestionario 0Q-10 (instrumento evaluado) es un cuestionario de 10 items con un puntaje total (máximo 40) indicador del nivel actual de malestar psicológico (distress, en inglés). El mayor puntaje total indica mayor malestar.

El DUKE-AD (instrumento de referencia) consta de 17 ítems y povee información del estado funcional, incluyendo ansiedad y depresión. El DUKE-AD tiene 7 ítems que reflejan el criterio de ansiedad y depresión según el DSM IV. Tiene un puntaje de 0 a 100 y resultados $>30$ indican ansiedad y/o depresión. El DUKE-AD se eligió como instrumento validado, usado en atención primaria para rastreo de ansiedad y depresión, para compararlo con el 0Q-10.

Se analizaron datos demográficos, reporte de hospitalizaciones y percepción del estado de salud. Esto último a través de una sola pregunta tomada del SF 36 y con un score entre 1 (peor) a 5 (excelente).

\section{Medición de resultados}

Se calculó la sensibilidad y especificidad* para distintos puntos de corte del 0Q-10 con el punto de corte del DUKE-AD para malestar/de- presión. Se definió sensibilidad como la habilidad del 0Q-10 para detectar malestar en pacientes que tienen criterio de ansiedad/depresión por DUKE-AD; y especificidad como la habilidad del 0Q-10 para clasificar pacientes que no tienen malestar por criterios de DUKE-AD. Se evaluó la correlación * entre el puntaje total de 0Q-10, puntajes 0Q-10 independientes (bienestar y malestar), escala específica de DUKE, factores sociodemográficos y percepción de salud.

\section{Resultados}

La media de edad fue 37 (DS 15), 73\% mujeres, 86\% de nivel educativo secundario. La media de la percepción de salud fue 2.7 (DS 1.1), 41 \% reportaron su salud como buena o excelente. La media del puntaje total de $0 Q$ 10 fue 13 (SD 7.05) y el tiempo estimado de respuesta fue $<10$ minutos.

\section{Sensibilidad y Especificidad del 0Q-10 usando el criterio del DUKE-AD} (ansiedad o depresión)

\begin{tabular}{lllllllll}
\hline OQ-10 score & 10 & 11 & 12 & 13 & 14 & 15 & 16 & 17 \\
\hline Basal\# & 0.55 & 0.55 & 0.55 & 0.55 & 0.55 & 0.55 & 0.55 & 0.55 \\
\hline VPP \# & 0.77 & 0.79 & 0.81 & 0.84 & 0.86 & 0.91 & 0.95 & 096 \\
\hline Especificidad & 0.69 & 0.74 & 0.78 & 0.84 & 0.88 & 0.94 & 0.97 & 0.98 \\
\hline Sensibilidad & 0.79 & 0.76 & 0.72 & 0.67 & 0.58 & 0.50 & 0.44 & 0.41 \\
\hline \# Basal: proporción de pacientes con angustia identificados correctamente \\
usando el DUKE-AD \\
VPP: valor predictivo positivo* proporción de pacientes con angustia correc- \\
tamente identificados por el 0Q-10 usando el criterio del DUKE -AD
\end{tabular}

La correlación entre el puntaje total del 0Q-10 y la subescala DUKE-AD fue $r=0.72(p<0.001)$; entre el puntaje 0Q-10 de bienestar y DUKE autoestima fue $r=0.61(p<0.001)$ y 0Q-10 y percepción de salud fue $r=0.41(p<0.01)$. Entre 0Q-10 de bienestar y DUKE -AD La correlación fue $r=0.53(p<0.001)$.

\section{Conclusiones}

El 0Q-10 puede ser utilizado como instrumento útil y breve de rastreo para medir malestar psicológico (distress) en atención primaria. Algunos ítems reflejan aspectos de bienestar y otros de angustia o depresión; lo cual resulta útil para el médico en el contexto de otros problemas del paciente.

\section{COMENTARIO}

Los problemas como depresión y angustia, son probablemente los más frecuentes en relación al estado psicológico de un paciente en la consulta ambulatoria. Aún así, diagnosticarlos no resulta sencillo. Existen otros estados, de dificultad o malestar, con síntomas físicos vagos que resultan más difíciles de identificar con un término preciso y que en ingles se denomina distress. Esto es un estado psicológico negativo, leve a severo, debido a multiples causas. Estos pacientes no cumplen criterios estrictos para un diagnóstico psiquiátrico de depresión o ansiedad. Algunos estudios indican que el distress tiene una prevalencia del $70 \%$ en la población de atención primaria. ${ }^{1}$

Quizás estos resultados ayudaron a plantear la pregunta de este estudio; y pensar así en algún instrumento que pudiera detectar estos estados que afectan la calidad de vida del paciente, y que también condicionan la evolución de ciertas patologías médicas.

Algunos aspectos para aclarar del trabajo son: las características evaluadas del instrumento: el punto de corte a utilizar; cuan conservador uno sería para detectar un estado de angustia o malestar leve? En esta muestra si el punto de corte a utilizar del 0Q-10 fuera de 12, casi el $50 \%$ de los pacientes serian identificados con angustia/malestar y con un punto de corte de 17 serían el $24 \%$. Este aspecto no esta muy claro y los autores plantean la utilización del punto de corte a criterio del médico, dependiendo del contexto y la población a analizar.

Es importante destacar que, al igual que otros instrumentos evaluados y validados para identificar diferentes patologías psiquiátricas y/o estado funcional de salud (MOS, SF36, PRIME MD, etc) ${ }^{2}$,el OQ-10 ha sido desarrollado en el ámbito de la atención primaria.

La correlación del 0Q-10 fue significativa con el DUKE-AD tanto en criterios de ansiedad y depresión como en percepción de salud y estado de bienestar. Uno podría pensar, entonces, que utilizar un instrumento rápido y simple como el 00-10 para identificar aspectos de la salud mental (p.ej. bienestar, ansiedad, etc.) facilitaría el proceso de atención de otras manifestaciones en la salud de nuestros pacientes.

Dra. Nora Gimpel Unidad de Medicina Familiar y Preventiva. Hospital Italiano de Buenos Aires. 